\title{
John P. H. Wilding (ed): Pharmacotherapy of obesity (Series: MDT-Milestones in Drug Therapy)
}

\author{
2008, 132 pp., Hardcover, Birkhäuser Verlag AG, Basel, Switzerland, \\ ISBN 978-3-7643-7138-8
}

\author{
Steven T. Russell
}

Published online: 24 December 2009

(c) Birkhäuser Verlag, Basel/Switzerland 2009

Over the last 10 years the treatment of obesity has become a major health issue worldwide rather than merely a cosmetic issue it once was and it has created a huge strain on healthcare budgets thought the industrialised countries. Obese patients have an impact on many areas of medicine, for example diabetes, cardiovascular disease, joint disease and respiratory disease. With this impact on healthcare, adipose tissue is now seen as not only a storage vessel but also as an endocrine organ in its own right involved in a number of metabolic diseases.

This book attempts to give a clear update of this obesity research and is readily accessible to healthcare professionals.

First Xavier Pi-Sunyer describes the need for medication in the treatment of obesity and discusses the jaded history of obesity treatments; there have been many attempts at treatment with only a few making any real impact, without unmanageable side effects. George Bray then discusses some more of the historical aspects of drug treatment.

Some of the key issues in the treatment of obesity are then discussed in detail such as energy balance where Joanne Harrold describes rational design for identifying usable treatments and the difficulties of extrapolation data from mouse to man; she also discusses the combinations of short- and long-term converging signals.

Lipase inhibitors are then described by John Wilding, in this case Orlistat, and its blockade of absorption of fat but the need for a focus on the promotion of a negative energy balance is also recognised.

S. T. Russell

Life and Health Sciences, Aston University, Aston Triangle,

Birmingham B4 7ET, UK

e-mail: s.t.russell1@aston.ac.uk
John then goes onto discuss sibutramine. He discusses preclinical and clinical pharmacology, particularly, the Sibutramine Cardiovascular Outcomes Trial (SCOUT), the results of which are released in 2009.

Future targets are also discussed in detail by Muhammad Khan and John Wilding with respect to the endocannabinoid system. Although the cannabinoid receptor antagonist rimonobant has been recently been withdrawn since publication of the book due to high suicide rates in some populations, it had seemed a good target as an appetite suppressant compared to thermoregulatory agents which increase energy expenditure.

Three broad areas are then discussed that are targets for drug development.

Chaudhri, Smith and Bloom describe some of the body's own peripheral and central signals in regulating appetite and feeding such as gut hormones. These gut hormones are many and play a vital physiological role in optimising gut function as an organ of digestion and absorption.

Finally John Clapham and Jon Arch discuss the influence of energy expenditure and substrate utilisation. The use of thermogenic drugs is discussed, for example, in stimulating the likes of fat oxidation. They also mention the role of $\beta_{3}$-adrenoceptor agonists as a possible therapeutic avenue. They then discuss in detail the biochemistry behind thermogenesis and some of the possible signal hormone mimics and intracellular targets, before discussing lipid synthesis and metabolism.

This is a welcome update to the field of obesity research and will be of use to healthcare professional and researchers alike. 